\title{
Percepción de estudiantes sobre la comunicación institucional universitaria
}

\section{Students' perception of university communication \\ Percepção dos alunos sobre a comunicação universitária}

Adriana Graciela Segura-Mariño, Pontificia Universidad Católica del Ecuador, Santo Domingo, Ecuador (agsegura@pucesd.edu.ec)

Manuel Fernández-Sande, Universidad Complutense de Madrid, Madrid, España (manuel.fernandez@ucm.es)

Francisco Javier Paniagua-Rojano, Universidad de Málaga, Málaga, España (fpaniagua@uma.es)

RESUMEN|El objetivo de esta investigación es evaluar la comunicación institucional de 21 universidades ecuatorianas desde la percepción de los estudiantes. Se aplicó una encuesta en línea con consistencia interna $(\omega=0,91)$ a 3540 alumnos y se valoró su percepción como estudiantes potenciales y actuales; los datos se analizaron mediantela prueba U de Mann-Whitney y el coeficiente $\tau$ de Kendall. Se destaca que el factor que más influyó para elegir la institución es el costo, que las universidades privadas gestionan mejor la relación con el alumnado y que la satisfacción sobre la comunicación está correlacionada con el compromiso que sienten los estudiantes actuales. Es indispensable que las instituciones de educación superior cuenten con equipos propios de comunicación y que valoren los canales en línea y offline, así como la percepción de padres/madres, ya que tienen una alta influencia en la decisión de sus hijos.

PALABRAS CLAVE: comunicación organizacional; universidad; educación superior; percepción estudiantil; marketing. 
ABSTRACT | The aim of this research is to evaluate the organizational communication of 21 Ecuadorian universities, from the audience's perception. An online survey with internal consistency $(\omega=0.91)$ was applied to 3,540 students. Their perception as potential and current students was valued, the data were analyzed using the Mann-Whitney Utest and the Kendall's c coefficient. It is evident that the factor that influenced the most the institution choice is cost, that private universities manage the relationship with students better, and that satisfaction with institutional communication is correlated with commitment that current students feel. It is essential that HEIs have their own communication departments and that they value online and offline channels, as well as the perception of parents, since they have a high influence on the decision of their children.

KEYWORDS: organizational communication; universities; higher education; student perception; marketing.

RESUMO | O objetivo desta pesquisa é avaliar a comunicação organizacional de 21 universidades equatorianas, a partir da percepção dos alunos. Foi aplicada uma pesquisa on-line com consistência interna $(\omega=0,91)$ a 3.540 estudantes, e sua percepção foi avaliada como estudantes potenciais e atuais; os dados foram analisados pelo teste U de Mann-Whitney e pelo coeficiente $\otimes$ de Kendall. Destacase que o fator que mais influenciou na escolha da instituição é o custo, que as universidades privadas melhor gerenciam o relacionamento com os alunos e que a satisfação com a comunicação está correlacionada com o comprometimento sentido pelos estudantes atuais. É essencial que as instituições de ensino superior tenham suas próprias equipes de comunicação e valorizem os canais online e offline, bem como a percepção dos pais e mães, pois têm grande influência na decisão dos filhos.

PALAVRAS-CHAVE: comunicação organizacional; universidade; ensino superior; percepção dos estudantes; marketing. 


\section{INTRODUCCIÓN}

Existen diversos estudios sobre el uso de medios con fines académicos, pero en el contexto institucional se ha investigado poco sobre las necesidades de comunicación de los estudiantes. Norteamérica y Europa lideran los rankings académicos; en consecuencia, hay escasas publicaciones sobre universidades latinoamericanas.

La comunicación interactiva ha hecho que sectores como la educación superior, que parecían seguros, se enfrenten a fuerzas competitivas (Khanna et al., 2014), debido a la presión por responder con recursos limitados a una demanda creciente (Alves \& Raposo, 2010). La comunicación en línea, al ser de acceso abierto, constituye una herramienta indispensable en un escenario lleno de incertidumbres para estudiantes actuales y potenciales, quienes necesitan analizar la institución por la cual optar, frente a la posibilidad de equivocarse en su decisión.

Internet promueve la competitividad académica, científica y social, contribuye a mejorar el posicionamiento, a conseguir más estudiantes y a consolidar equipos de investigación (Ortega \& Aguillo, 2009), pero eso si es que la comunicación es estratégica para, con base en la investigación, satisfacerlas necesidades de información de sus stakeholders y proyectar una imagen consecuente con su misión (Veas, 2015).

Los grupos de interés son principalmente futuros y actuales estudiantes. Para atraerlos y fidelizarlos, las universidades tienen que conocer sus expectativas (García-García, 2018). A nivel global, 61\% de internautas cursan estudios universitarios y son quienes más se conectan a diario, alrededor de cuatro horas y 15 min (GlobalWebIndex, 2019). Esto ha generado el interés por realizar investigaciones sobre el alumnado, en su calidad de estudiantes potenciales y actuales.

En el caso de los estudiantes potenciales, existe literatura sobre el nivel de dificultad para elegir la universidad, factores institucionales, de comunicación y de recomendaciones que han influido en dicha elección, entre otros, como la importancia que otorgan a los canales de comunicación institucional durante el proceso de decisión. En cuanto al alumnado actual, se abordan los temas que son importantes para ellos, y cómo valoran la gestión de la comunicación que sus universidades despliegan en la búsqueda por fortalecer su relación con ellos.

\section{Factores asociados a la selección de universidades por parte de estudiantes potenciales}

Conocer la percepción del alumnado potencial sobre la comunicación organizacional de las universidades permite una planificación de medios personalizada y efectiva (Goodrich et al., 2018). Aunque en algunas pesquisas se ha procurado elaborar una lista definitiva de factores que influyen en la decisión de los estudiantes sobre qué universidad elegir, no existen resultados homogéneos, debido al evidente contraste entre países (Hemsley-Brown \& Oplatka, 2015). 
Por ejemplo, en Inglaterra, las oportunidades de empleo motivaron más que la vocación para elegir la institución (Maringe, 2006). De igual manera, el factor que más influyó en estudiantes de universidades públicas de Valencia (España) fue la expectativa laboral (Fayos et al., 2011). Por su parte, las personas matriculadas en una universidad pública portuguesa estimaron que era más importante la cercanía entre su domicilio y la localidad de la institución (Simões \& Soares, 2010).

En otros contextos, los estudiantes de una universidad del Medio Oeste de Estados Unidos valoraban más la asesoría académica y la efectividad educativa según Elliott \& Healy (2001) - quienes sugerían incluir el prestigio o el precio en estudios posteriores. Al respecto, Casidy y Wymer (2018) definieron tres perfiles de estudiantes en Australia, en función de factores psicográficos: luchadores, triunfadores modestos, e innovadores que buscan prestigio. Los dos últimos demostraron una actitud más positiva hacia su universidad, en tanto que los luchadores, si pudieran volver a elegir, elegirían una institución más prestigiosa.

Peruta y Shields (2016) señalan que las universidades utilizan las redes sociales para obtener información de los potenciales estudiantes durante el proceso de decisión, fundamentalmente Facebook. Para Hayes y sus colegas (2009) era lógico suponer que el uso de redes haría que las IES se interesaran en utilizarlas para el reclutamiento. No obstante, en Estados Unidos los medios en línea no han sustituido a las visitas al campus o a los sitios web (Shields \& Peruta, 2018). En Reino Unido, el alumnado considera que las fuentes de información más importantes para decidir fueron el cibersitio de Universities \& Colleges Admissions Service y los portales universitarios. En cuanto a los medios sociales, los perciben como canales divertidos y conversacionales, donde pueden encontrar opiniones, pero reconocen que no son idóneos para encontrar contenidos pormenorizados y tomar una decisión (Gibbs \& Dean, 2015).

De manera similar, para el alumnado de 12 provincias de los Países Bajos, los medios sociales fueron los canales menos utilizados para informarse y decidir el programa y la institución; aunque el 95,1\% había confirmado tener una cuenta activa, las recomendaciones de familiares/amistades ejercieron más influencia (Constantinides \& Zinck Stagno, 2011). Los medios que prefirieron los estudiantes de una universidad estadounidense fueron las visitas al campus, los portales y las recomendaciones de familiares/amistades (Goodrich et al., 2018). El medio más consultado por estudiantes de una universidad portuguesa fue el cibersitio, seguido por la recomendación de otros alumnos y profesores (Simões \& Soares, 2010).

Por su parte, más del 40\% de los estudiantes de una universidad griega confirmó no haber buscado información en canales institucionales, sino consultar la opinión de grupos de referencia (Menon et al., 2007). Los universitarios galeses habrían preferido un mayor uso de recursos digitales cuya fuente hubiese sido el propio 
alumnado (Moogan, 2011). En Inglaterra han confiado más en las recomendaciones de amistades/familiares o personas a quienes no conocían, pero por quienes sentían afinidad, que en información facilitada por la propia universidad (Slack et al., 2014). El boca a boca ha sido importante para estudiantes potenciales en Turquía, pues los consejos de amistades/familiares fueron más efectivos que los canales convencionales. Özdemir et al., (2016) sostienen que la satisfacción y la comunicación transparente generan la recomendación, por lo que es indispensable que los servicios de educación superior que ofrecen las universidades respondan a las expectativas que los alumnos tienen sobre ellas.

La comunicación eficaz de la responsabilidad social universitaria, entendida como una política de mejora continua para cumplir su misión social (Vallaeys, 2008) constituye otro factor de importante influencia en la elección de la universidad (Erazo-Coronado et al., 2020).

\section{Factores asociados a la imagen de la universidad en el caso de los estudiantes}

Las IES tienen que investigar qué es importante para su alumnado, generar expectativas y cumplirlas, puesto que el medio de comunicación más eficaz surge de estudiantes satisfechos (Elliott \& Healy, 2001). Además, la educación superior requiere de una relación participativa e interactiva por parte de las universidades y de sus estudiantes para evitar la deserción estudiantil (Alves \& Raposo, 2010; Helgesen, 2008).

Para el alumnado de la Escuela Universitaria de Ålesund, en Noruega, la satisfacción y percepción sobre la reputación institucional influyen en su lealtad (Helgesen, 2008). De manera muy similar, los estudiantes de universidades públicas portuguesas mencionaron que la imagen, las creencias y las impresiones que existen sobre su institución es lo que más influye en su satisfacción y lealtad (Alves \& Raposo, 2010). Para los estudiantes de una universidad chilena, es el compromiso lo que más influye en su lealtad; sin embargo, como explican RojasMéndez et al. (2009), la calidad de la educación que los estudiantes perciben influye primero en su grado de satisfacción, que a su vez influye en la confianza y esta, en el compromiso. Gallegos y Vasquez (2019) confirman la vigencia de esta secuencia de satisfacción, confianza y compromiso, que culmina con la lealtad de los estudiantes. En dos estudios realizados en Brasil se sostiene que la confianza y la lealtad son fundamentales para conseguir más participación estudiantil (Carvalho \& de Oliveira Mota, 2010; Sampaio et al., 2012). Sumartias y Nuraryo (2017), en el ámbito del análisis de la comunicación de las universidades privadas de Indonesia, constatan de forma empírica la influencia directa de los elevados niveles de satisfacción y de una buena imagen de la marca universitaria en la lealtad de los estudiantes, y la capacidad de esta marca para generar comunicación positiva. 
Todos estos factores constituyen predictores críticos del desarrollo de una transmisión boca en boca positiva, a la que otorgan una importancia estratégica para fidelizar a los estudiantes y lograr que prosigan en la institución universitaria, cursando estudios de posgrado (Rehman et al., 2020).

En efecto, la calidad académica, la reputación institucional y el servicio de educación superior que en conjunto ofrecen las universidades influyen en la satisfacción y lealtad, sobre lo que hay numerosos estudios. No obstante, se conoce poco acerca del nivel de satisfacción que los alumnos tienen con respecto a cómo las IES cuidan su relación con ellos, es decir cómo gestionan la comunicación institucional (Aitken, 1982; Rodríguez \& Santamaría, 2012).

Por ejemplo, para los estudiantes portugueses la calidad de los sitios web no es alta, y consideran que sus universidades no utilizan los cibersitios como herramienta de comunicación para reforzar la relación, conseguir su lealtad y responder a sus necesidades de información (Vera Silva \& Gouveia Rodrigues, 2012).

Encuanto aredes sociales, y segúnla percepción de estudiantes y alumni deescuelas de administración en India, estas deben mejorar sus estrategias de comunicación, ya que son canales donde alumnos y graduados pueden ayudar a difundir información y mejorar el valor de marca (Kahnna et al., 2014). Se han comprobado los efectos positivos de Instagram en la estrategia de comunicación digital de las universidades de Indonesia para reforzar el compromiso de sus estudiantes (Kurniawan et al., 2020). El tipo de contenido de las publicaciones institucionales en esta red social resulta determinante para generar este compromiso e incentivar la participación de los estudiantes (Bonilla et al., 2020). Se ha detectado que las redes mejoran la calidad de la relación entre una universidad pública del Este de Estados Unidos y sus estudiantes (Clark et al., 2017). Sin embargo, en la Universidad de Sunshine Coast (Australia) los estudiantes nunca o rara vez las utilizan para interactuar en el contexto offline con la comunidad universitaria; esto influye en que no se sientan totalmente conectados con ella (Sutherland et al., 2018). La orientación al mercado y la estrategia de marca de las universidades se han identificado como variables con impacto positivo y muy significativo en los índices de satisfacción, lealtad y comportamiento comunicacional de los estudiantes australianos (Casidy, 2014).

Considerando que existen escasos estudios sobre cómo los alumnos perciben la comunicación institucional de las universidades, desde su postura como estudiantes potenciales y actuales, especialmente en Latinoamérica, el objetivo de este trabajo es evaluar la comunicación universitaria desde la percepción de la audiencia y presentar los resultados de una metodología empleada en Ecuador, que es adaptable a los países de la región. Para tal fin, se plantearon nueve preguntas de investigación, con base en la revisión de la literatura. 
Preguntas de investigación para estudiantes potenciales (1-3):

PI1. ¿Qué nivel de dificultad tuvieron los estudiantes para elegirla universidad? PI2. ¿Qué nivel de importancia tienen los factores institucionales, de comunicaciones de marketing y de recomendación para elegir la universidad?

PI3. ¿Qué nivel de importancia habrían tenido los canales de comunicación institucional durante el proceso de decisión?

Preguntas de investigación para estudiantes (4-9):

PI4. ¿Qué tan importante es para los estudiantes estar informados sobre temas de sus universidades?

PI5. ¿Qué tan satisfactoria es la comunicación que las instituciones dirigen a sus estudiantes sobre temas universitarios?

PI6. ¿Cómo valoran los canales de comunicación institucional?

PI7. ¿Qué nivel de satisfacción sienten sobre la comunicación universitaria desplegada en los canales en línea?

PI8. ¿Qué nivel de compromiso sienten con sus universidades?

PI9. ¿Existe correlación entre el nivel de satisfacción con la comunicación institucional y el nivel de compromiso que sienten con sus universidades?

\section{METOdOLOGÍA}

\section{Población y muestra}

En estudios sobre la percepción estudiantil, Shields y Peruta (2018) sugieren incluir instituciones de educación superior privadas y públicas para que los resultados sean generalizables; por consiguiente, se aplicó el muestreo estratificado. Cabe mencionar que en Ecuador la clasificación de las instituciones de educación superior se determina en función del financiamiento, en cuatro tipos: particulares que reciben fondos del Estado, particulares autofinanciadas públicas nacionales, y públicas que operan en el país bajo acuerdos y convenios internacionales.

Se consultó la población estudiantil (621.210) del año 2019 en los portales web de las 57 universidades ecuatorianas que ofrecen carreras de grado mediante la información pública disponible en la rendición de cuentas de las universidades. Posteriormente se calculó la muestra con un nivel de confianza de 97\% y error de muestreo de 3\%. Se obtuvo un total de 1306 estudiantes (493 que corresponderían al estrato de instituciones privadas y 813 al de instituciones públicas). 
La meta se superó, ya que se consiguieron 3540 encuestas, 620 de universidades privadas y 2920 de universidades públicas.

\section{Proceso}

Se enviaron comunicados hasta por tres ocasiones a los 57 rectores, para dar a conocer el objetivo de la investigación y solicitar que se compartiera la encuesta a sus estudiantes mediante los canales institucionales en línea. Se dio a conocer que los resultados de la investigación se verían reflejados en un artículo científico, que sería compartido con ellos una vez publicado. Asimismo, quedó por escrito el compromiso de mantener el anonimato de los participantes, y la no divulgación de sus datos personales. Del total de universidades, 42 autoridades no respondieron a la petición, 14 respondieron positivamente y una dio respuesta negativa. Cabe mencionar que aunque la mayoría de rectores no contestaron y aunque se desconocen sus razones, finalmente participaron 21 universidades.

En Ecuador existen dos periodos académicos por año, que oscilan entre abrilagosto y octubre-febrero. Las respuestas se receptaron en enero de 2020, para obtener respuestas a las preguntas de satisfacción de los alumnos de primer nivel. Su participación era voluntaria y al introducir el instrumento, además de agradecer su predisposición a participar, se indicó el tiempo estimado de respuesta (entre 5 y 7 minutos), se dio a conocer el objetivo de la investigación y se expresó que su identidad no sería revelada. Adicionalmente, se facilitaron los datos de contacto de las personas responsables del estudio, incluyendo correos electrónicos institucionales.

\section{Instrumento}

Para diseñar el cuestionario fueron fundamentales los aportes de estudios similares (Casidy \& Wymer, 2018; Constantinides \& Zinck Stagno, 2011; Elliott \& Healy, 2001; Helgesen, 2008; Maringe, 2006; Özdemir et al., 2016; Shields \& Peruta, 2018). Más adelante se ofrecen detalles. La encuesta se elaboró en un formulario de Google.

Para determinar el nivel de comprensión de las preguntas y qué elementos se podían añadir o eliminar se entrevistó a siete estudiantes que no formaban parte de la muestra. Luego de ello, con el mismo propósito se procedió a encuestar a 40 estudiantes -que tampoco formaban parte de la muestra-con su correspondiente cálculo de fiabilidad del instrumento. Es importante mencionar que la fiabilidad se volvió a calcular una vez alcanzadas las 3540 respuestas, mediante el coeficiente de omega, óptimo para estudios sociales que incluyen ítems con escala Likert (Deng \& Chan, 2017; Viladrich et al., 2017), porque presenta menos riesgos que el alfa de Cronbach de sobreestimar o subestimar la fiabilidad (Dunn et al., 2014). Se obtuvo en promedio $\omega=0,91$ (tabla 1 ). 
El instrumento contiene 73 ítems distribuidos en tres secciones principales (tabla 1). La sección 1 contiene variables numéricas, dicotómicas y politómicas acerca de datos generales; las secciones 2 y 3 contienen variables categóricas tipo Likert sobre la percepción antes de haber ingresado a la universidad y sobre la percepción como estudiantes actuales, respectivamente. De los elementos de otros autores que han sido adaptados al instrumento, los trabajos de Constantinides y Zinck Stagno (2011), de Maringe (2006), de Özdemir et al., (2016) y de Shields y Peruta (2018) fueron clave para la segunda sección; los aportes de Elliott y Healy (2001) y de Helgesen (2008) lo fueron para la tercera. En cuanto al estudio de Casidy y Wymer (2018), fue clave para la segunda y tercera sección.

Se crearon seis índices. Para la segunda sección sobre estudiantes potenciales: índice de factores institucionales (IFI), índice de factores de comunicaciones de marketing (IFCM) e índice de factores de recomendación (IFR). Para la tercera sección sobre estudiantes actuales: índice de satisfacción de temas (IST), índice de satisfacción sobre canales institucionales (ISCI) e índice del nivel de compromiso universitario (INCU). El cálculo para crear cada índice se basó en la propuesta de Berganza y sus colegas (2017), donde $\mathrm{I}=\Sigma(\mathrm{x} 1, \mathrm{x} 2 \ldots \mathrm{xn}) / \mathrm{n}$. Es decir que I=índice, $\mathrm{x}=$ variables $\mathrm{y} \mathrm{n}=$ número de variables.

1. Datos generales

Edad

Sexo

Universidad/tipo de financiamiento - pública o privada-

Duración de la carrera en años

Año de estudio

Área de conocimiento de la carrera según la UNESCO

2. Percepción antes de haber ingresado a la universidad

Nivel de dificultad para elegir la universidad

\section{Importancia de factores institucionales para elegir la universidad} Índice de factores institucionales (IFI)

Reputación institucional

Posición en rankings

Becas/facilidades de pago

Costos de estudios

Localidad/ubicación 
Importancia de factores de comunicaciones de marketing para elegir la universidad Índice de factores de comunicaciones de marketing (IFCM)

Anuncios en televisión

Anuncios en prensa

Anuncios en radio

Publicidad que aparecía en sitios web ajenos a la universidad

Publicidad que aparecía en redes sociales

Visita/s que la universidad hizo a su colegio

Ferias de universidades

\section{Importancia de factores de recomendación para elegir la universidad} Índice de factores de recomendación (IFR)

Estudiantes de la universidad

Graduados en la universidad

Profesores del colegio o de la secundaria

Asesores vocacionales

Amistades/conocidos

Foros o comunidades en Internet

Familiares

Padres/madres

Importancia de canales de comunicación institucional como fuentes de información para estudiantes potenciales

Chat en el sitio web

Formulario de contacto disponible en el sitio web

Chat en alguna red social de la universidad

Correo electrónico de la universidad

Visitas a las redes sociales de la universidad

Llamadas telefónicas

Sitio web

WhatsApp de la universidad

Visitas a la universidad

Prospectos/folletos

3. Percepción como estudiante universitario

\section{Importancia de estar informados sobre los siguientes temas}

Institucionales: noticias/información de la universidad en general

De interés o desarrollo social, días festivos, días conmemorativos

Académicos: noticias/información de grado, posgrado o educación continua

Investigación y desarrollo

Eventos: institucionales, sociales, deportivos 
Satisfacción sobre la comunicación que las universidades dirigen a sus estudiantes acerca de los siguientes temas

Índice de satisfacción de temas (IST)

Académicos: noticias/información de grado, posgrado o educación continua

De interés o desarrollo social, días festivos, días conmemorativos

Eventos: institucionales, sociales, deportivos

Institucionales: noticias/información de la universidad en general

Investigación y desarrollo

Conveniencia de los canales de comunicación universitaria como fuentes de información para estudiantes actuales

Plataforma académica de la universidad
Correo electrónico personal
Correo electrónico institucional
Sitio web
Revista/boletín institucional
Redes sociales
Llamada telefónica
WhatsApp

Frecuencia con la que los estudiantes actuales consultan los canales en línea universitarios

YouTube

Instagram

Twitter

Facebook

Sitio web

Correo electrónico institucional

Satisfacción sobre la comunicación que las universidades dirigen a sus estudiantes a través de los canales en línea

Índice de satisfacción sobre canales institucionales (ISCI)

Instagram

YouTube

Twitter

Facebook

Sitio web

Correo electrónico institucional

Nivel de compromiso: nivel de acuerdo/desacuerdo con las siguientes afirmaciones Índice del nivel de compromiso universitario (INCU)

Recomendaría a otras personas estudiar en esta universidad

Si pudiera volver a elegir, elegiría la misma universidad

Elegiría estudiar un posgrado en la misma universidad

La universidad se preocupa por hacerme sentir parte de la comunidad universitaria

La universidad me envía información oportunamente

Me gusta compartir información de la universidad en mis redes sociales

Tabla 1. Instrumento

Fuente: Elaboración propia 


\section{RESULTADOS}

\section{Datos generales}

En Ecuador hay 57 universidades que ofrecen estudios de grado; 27 privadas y 30 públicas. Participaron estudiantes de 21 instituciones; 10 privadas y 11 públicas. De las 3540 encuestas, 620 provienen de universidades privadas y 2920, de universidades públicas. Los datos se analizaron en el programa estadístico SPSS.

Los encuestados tienen entre 17 y 67 años, la media es 22 y la desviación típica 4,29. Participaron 1658 (46,8\%) hombres y 1882 (53,2\%) mujeres, matriculados en carreras que duran entre 3 y 5 años, estudiantes de todos los niveles y de las 10 áreas del conocimiento, según el Instituto de Estadística de la UNESCO (2014).

\section{Percepción antes de haber ingresado a la universidad}

En esta sección las variables son ordinales y se analizaron mediante la prueba U de Mann-Whitney para comparar los resultados según los estratos. En una pregunta de transición, siendo 1 nada difícil y 5 muy difícil, se detectó que la dificultad para elegir la universidad fue baja, se obtuvo de media 2,41 y desviación típica, 1,19. El nivel de dificultad difiere significativamente para estudiantes de universidades privadas $M=1999,90$ y de universidades públicas $M=1721,79$; $\mathrm{U}=762974,000, \mathrm{z}=-6,369, \mathrm{p}=0,000$.

\section{Factores que influyen en la elección de la universidad}

Se analizó la importancia de 20 factores en la elección de la universidad. Se determinó que en siete existen diferencias significativas según el tipo de institución, sobre los cuales se detalla el rango promedio (tabla 2). Los costos y las becas/facilidades de pago influyeron más en estudiantes de instituciones privadas. En cambio, la posición en rankings, los anuncios en televisión, prensa y radio influyeron más en estudiantes de IES públicas. El factor institucional que más influyó es el costo; el de marketing que más influyó es la publicidad en redes sociales, y el de recomendación que más influyó es la opinión de padres/madres.

En función de los primeros tres índices creados, se observó que durante el proceso de decisión pesan más los factores institucionales, después los de recomendación y luego los de comunicaciones de marketing. Sobre 5 puntos, IFI=3,82; IFR=3,34; IFCM=2,97, existen diferencias significativas únicamente en el IFI, para estudiantes de universidades privadas $M=1846,96$ y de universidades públicas $\mathrm{M}=1754,27 ; \mathrm{U}=857797, \mathrm{z}=-2,057, \mathrm{p}=0,039$.

\section{Canales de comunicación institucional para estudiantes potenciales}

Se estableció qué tan importante habría sido encontrar información en diez medios institucionales de comunicación durante el proceso de decisión; en la tabla 3 se presentan los canales de mayor a menor importancia. No existen diferencias significativas según los estratos, $\mathrm{p}>0,05$. 


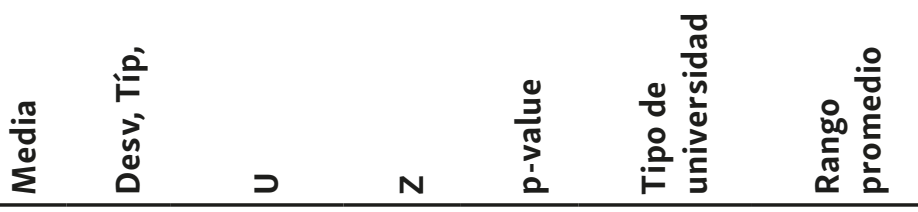

Importancia de factores institucionales para elegir la universidad Índice de factores institucionales (IFI)

\begin{tabular}{l|c|c|c|c|c|c|c|}
\hline Costos de estudios & 4,07 & 1,12 & 839671,5 & $-3,037$ & 0,002 & Privada & 1876,19 \\
\hline Localidad/ubicación & 3,95 & 1,21 & & & & & \\
\hline Reputación institucional & 3,82 & 1,15 & & & & & \\
\hline Becas/facilidades de pago & 3,64 & 1,29 & 696272,5 & $-9,366$ & 0,000 & Privada & 2107,48 \\
\hline Posición en rankings & 3,60 & 1,25 & 855619 & $-2,231$ & 0,026 & Pública & 1698,95 \\
\hline
\end{tabular}

Importancia de factores de comunicaciones de marketing para elegir la universidad Índice de factores de comunicaciones de marketing (IFCM)

\begin{tabular}{|c|c|c|c|c|c|c|c|}
\hline $\begin{array}{l}\text { Publicidad que aparecía en } \\
\text { redes sociales }\end{array}$ & 3,39 & 1,31 & & & & & \\
\hline Ferias de universidades & 3,27 & 1,42 & & & & & \\
\hline $\begin{array}{l}\text { Publicidad que aparecía } \\
\text { en sitios web ajenos a la } \\
\text { universidad }\end{array}$ & 3,19 & 1,35 & & & & & \\
\hline $\begin{array}{l}\text { Visita/s que la universidad } \\
\text { hizo a su colegio }\end{array}$ & 2,95 & 1,46 & & & & & \\
\hline \multirow{2}{*}{ Anuncios en televisión } & \multirow[b]{2}{*}{2,76} & \multirow[b]{2}{*}{1,35} & \multirow{2}{*}{813148} & \multirow{2}{*}{$-4,078$} & \multirow{2}{*}{0,000} & Privada & 1622,03 \\
\hline & & & & & & Pública & 1802,02 \\
\hline \multirow{2}{*}{ Anuncios en prensa } & \multirow{2}{*}{2,71} & \multirow{2}{*}{1,34} & \multirow{2}{*}{805403} & \multirow{2}{*}{$-4,424$} & \multirow{2}{*}{0,000} & Privada & 1609,54 \\
\hline & & & & & & Pública & 1804,68 \\
\hline \multirow{2}{*}{ Anuncios en radio } & \multirow{2}{*}{2,51} & \multirow{2}{*}{1,33} & \multirow{2}{*}{849252,5} & \multirow{2}{*}{$-2,492$} & \multirow{2}{*}{0,013} & Privada & 1680,26 \\
\hline & & & & & & Pública & 1789,66 \\
\hline
\end{tabular}

Importancia de factores de recomendación para elegir la universidad Índice de factores de recomendación (IFR)

\begin{tabular}{|c|c|c|c|c|c|c|c|}
\hline Padres/madres & 3,98 & 1,22 & & & & & \\
\hline Familiares & 3,54 & 1,28 & & & & & \\
\hline $\begin{array}{l}\text { Estudiantes de la } \\
\text { universidad }\end{array}$ & 3,39 & 1,35 & & & & & \\
\hline $\begin{array}{l}\text { Graduados en la } \\
\text { universidad }\end{array}$ & 3,38 & 1,40 & & & & & \\
\hline Amistades/conocidos & 3,25 & 1,25 & & & & & \\
\hline \multirow{2}{*}{$\begin{array}{l}\text { Profesores del colegio o de } \\
\text { la secundaria }\end{array}$} & \multirow{2}{*}{3,23} & \multirow{2}{*}{1,34} & \multirow{2}{*}{828507} & \multirow{2}{*}{$-3,404$} & \multirow{2}{*}{0,001} & Privada & 1646,8 \\
\hline & & & & & & Pública & 1796,76 \\
\hline $\begin{array}{l}\text { Foros o comunidades en } \\
\text { Internet }\end{array}$ & 3,01 & 1,37 & & & & & \\
\hline Asesores vocacionales & 2,93 & 1,38 & & & & & \\
\hline
\end{tabular}

Nota: los ítems de cada grupo de factores están ordenados de mayor a menor, en función de la media.

Tabla 2. Factores que influyen en la elección de la universidad

Fuente: Elaboración propia. 


\begin{tabular}{ccc} 
Canal & Media & Desv, Típ, \\
\hline Sitio web & 3,98 & 1,061 \\
\hline Visitas a la universidad & 3,97 & 1,124 \\
\hline Visitas a las redes sociales & 3,86 & 1,145 \\
\hline Formulario de contacto en el sitio web & 3,78 & 1,134 \\
\hline Correo electrónico de la universidad & 3,71 & 1,188 \\
\hline Chat en el sitio web & 3,58 & 1,231 \\
\hline Prospectos/folletos & 3,55 & 1,226 \\
\hline Chat en alguna red social de la universidad & 3,52 & 1,251 \\
\hline Llamadas telefónicas & 3,46 & 1,262 \\
\hline WhatsApp de la universidad & 3,37 & 1,349 \\
\hline
\end{tabular}

Tabla 3. Importancia de canales institucionales para estudiantes potenciales

Fuente: Elaboración propia.

\section{Percepción como estudiante universitario}

En esta sección los análisis se realizaron mediante la prueba U de Mann-Whitney $y$, en la última parte, mediante el coeficiente $\tau$ de Kendall. Para el alumnado actual es prioritario mantenerse informados sobre temas de investigación, académicos, institucionales, de interés social y eventos, en ese orden. No existen diferencias significativas entre tipos de universidades, $p>0,05$ (tabla 4). Sobre dichos temas, expresaron qué tan satisfactoria es la comunicación que las universidades dirigen. De mayor a menor el orden es: de investigación, interés social, eventos, académicos e institucionales. Existen diferencias significativas en 4 de los 5 temas. Cabe señalar que en función del IST existen diferencias significativas; para estudiantes de universidades privadas $M=1886,80$ y de universidades públicas $M=1745,81$, $\mathrm{U}=833091,000, \mathrm{z}=-3,157, \mathrm{p}=0,002$.

Se estableció en quémedida consideran convenientes ocho medios institucionales. Existen diferencias significativas en dos: correo institucional y correo personal. Expresaron con qué frecuencia consultan 6 canales en línea universitarios -fue necesario verificar en 57 portales que las IES los utilizaran-. En la tabla 4 se observa que el correo es consultado con mayor frecuencia. Existen diferencias significativas en el uso de cuatro canales: 1 correo, el sitio web e Instagram son consultados con más frecuencia por estudiantes de IES privadas, y Facebook por estudiantes de IES públicas. Sobre los canales, expresaron qué tan satisfactorio es el uso que las universidades hacen de ellos. El orden es el mismo que el de la frecuencia. Existen diferencias significativas en la satisfacción del uso de cuatro canales; los 
estudiantes de IES privadas se sienten más satisfechos que los de IES públicas con el uso que estas hacen del correo, sitio web, Instagram y Twitter (tabla 4). Esto es consecuente con que existan diferencias significativas en función del ISCI; para estudiantes de universidades privadas $M=1921,66$ y de universidades públicas $\mathrm{M}=1738,40, \mathrm{U}=811478,000, \mathrm{z}=-4,065, \mathrm{p}=0,000$.

Los estudiantes expresaron su nivel de acuerdo con seis afirmaciones. En función del índice del nivel de compromiso universitario se detectó que el nivel es alto, sobre 5 puntos INCU=3,85. No existen diferencias entre el INCU según los estratos, $p>0,05$, pero al realizar la prueba con cada afirmación se observaron diferencias en cuatro de ellas. Así, los estudiantes de universidades privadas expresan más acuerdo con que estas envían información oportunamente y que se preocupan por hacerles sentir parte de la comunidad universitaria. Los alumnos de universidades públicas expresan más acuerdo con que volverían a elegirla y con que estudiarían un posgrado en la misma institución (tabla 4).

Mediante el coeficiente $\tau$ de Kendall se detectó que el INCU está correlacionado con el IST; $\tau=0,497, p=0,000$. Igualmente, el INCU está correlacionado con el ISCI; $\tau=0,459, p=0,000$. Al realizar los análisis en función del año de estudio, se observó que si bien $\mathrm{p}<0,05$, las correlaciones son muy bajas o cercanas a cero ( $\mathrm{p}$-value entre 0,000 y 0,190), por lo tanto no conviene dar por sentado aquello. De igual manera, al analizar según la edad mediante la correlación de Spearman.

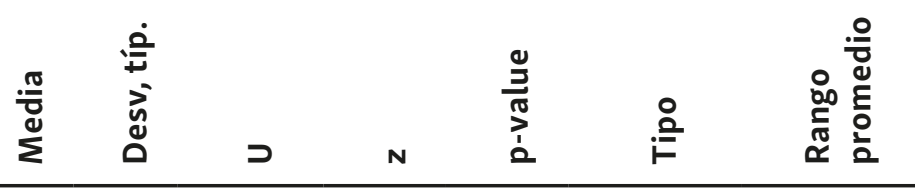

Importancia de estar informados sobre los siguientes temas

\begin{tabular}{l|l|l|l|l|l|l|l}
\hline Investigación y desarrollo & 4,46 & 0,79 & & & & & \\
\hline $\begin{array}{l}\text { Académicos: noticias/ } \\
\text { información de grado, } \\
\text { posgrado o educación } \\
\text { continua }\end{array}$ & 4,43 & 0,81 & & & & & \\
\hline $\begin{array}{l}\text { Institucionales: noticias/ } \\
\text { información de la } \\
\text { universidad en general }\end{array}$ & 4,31 & 0,87 & & & & & \\
\hline $\begin{array}{l}\text { De interés o desarrollo } \\
\text { social, días festivos, días } \\
\text { conmemorativos }\end{array}$ & 4,16 & 0,94 & & & & \\
\hline $\begin{array}{l}\text { Eventos: institucionales, } \\
\text { sociales, deportivos }\end{array}$ & 4,14 & 0,97 & & & & \\
\hline
\end{tabular}


Satisfacción sobre la comunicación que las universidades dirigen a sus estudiantes acerca de los siguientes temas

Índice de satisfacción de temas (IST)

\begin{tabular}{l|l|l|l|l|l|l|l}
\hline Investigación y desarrollo & 3,89 & 1,08 & & & & & \\
\hline $\begin{array}{l}\text { De interés o desarrollo } \\
\text { social, días festivos, días } \\
\text { conmemorativos }\end{array}$ & 3,85 & 1,03 & 849447,5 & $-2,539$ & 0,011 & Privada & 1860,42 \\
\hline $\begin{array}{l}\text { Eventos: institucionales, } \\
\text { sociales, deportivos }\end{array}$ & 3,82 & 1,07 & 848443,5 & $-2,58$ & 0,01 & Pública & 1751,41 \\
\hline $\begin{array}{l}\text { Académicos: noticias/ } \\
\text { información de grado, } \\
\text { posgrado o educación } \\
\text { continua }\end{array}$ & 3,81 & 1,06 & 825966,5 & $-3,598$ & 0,000 & Pública & 17562,04 \\
\hline $\begin{array}{l}\text { Institucionales: noticias/ } \\
\text { información de la } \\
\text { universidad en general }\end{array}$ & 3,79 & 1,06 & 821235 & $-3,815$ & 0,000 & Pública & 1743,37 \\
\hline
\end{tabular}

Conveniencia de los canales de comunicación universitaria como fuentes de información para estudiantes actuales

\begin{tabular}{l|c|l|l|l|l|l|l}
\hline Redes sociales & 4,32 & 0,91 & & & & & \\
\hline $\begin{array}{l}\text { Plataforma académica de } \\
\text { la universidad }\end{array}$ & 4,26 & 0,95 & & & & & \\
\hline $\begin{array}{l}\text { Correo electrónico } \\
\text { institucional }\end{array}$ & 4,20 & 0,99 & 855696,5 & $-2,324$ & 0,02 & Privada & 1850,34 \\
\hline $\begin{array}{l}\text { Correo electrónico } \\
\text { personal }\end{array}$ & 4,17 & 1,04 & 852824 & $-2,454$ & 0,014 & Privadica & 1753,55 \\
\hline \begin{tabular}{l} 
Sitio web \\
\hline WhatsApp
\end{tabular} & 4,14 & 1,01 & & & & & 1854,98 \\
\hline $\begin{array}{l}\text { Revista/boletín } \\
\text { institucional }\end{array}$ & 4,00 & 1,14 & & & & & 1752,56 \\
\hline \begin{tabular}{l} 
Llamada telefónica \\
\hline
\end{tabular} & 3,69 & 1,20 & & & & & \\
\hline
\end{tabular}

Frecuencia con la que los estudiantes actuales consultan los canales en línea universitarios

\begin{tabular}{|c|c|c|c|c|c|c|c|}
\hline \multirow{2}{*}{$\begin{array}{l}\text { Correo electrónico } \\
\text { institucional }\end{array}$} & \multirow{2}{*}{3,96} & \multirow{2}{*}{1,06} & \multirow{2}{*}{814623,5} & \multirow{2}{*}{$-4,124$} & \multirow{2}{*}{0,000} & Privada & 1916,59 \\
\hline & & & & & & Pública & 1739,48 \\
\hline \multirow{2}{*}{ Sitio web } & \multirow{2}{*}{3,77} & \multirow{2}{*}{1,04} & \multirow{2}{*}{833295,5} & \multirow{2}{*}{$-3,247$} & \multirow{2}{*}{0,001} & Privada & 1886,48 \\
\hline & & & & & & Pública & 1745,88 \\
\hline \multirow{2}{*}{ Facebook } & \multirow{2}{*}{3,66} & \multirow{2}{*}{1,19} & \multirow{2}{*}{842649} & \multirow{2}{*}{$-2,802$} & \multirow{2}{*}{0,005} & Privada & 1669,61 \\
\hline & & & & & & Pública & 1791,92 \\
\hline \multirow{2}{*}{ Instagram } & \multirow{2}{*}{2,90} & \multirow{2}{*}{1,46} & \multirow{2}{*}{752486,5} & \multirow{2}{*}{$-6,756$} & \multirow{2}{*}{0,000} & Privada & 2016,81 \\
\hline & & & & & & Pública & 1718,2 \\
\hline YouTube & 2,74 & 1,43 & & & & & \\
\hline Twitter & 2,21 & 1,33 & & & & & \\
\hline
\end{tabular}


Satisfacción sobre la comunicación que las universidades dirigen a sus estudiantes a través de los canales línea

Índice de satisfacción sobre canales institucionales (ISCI)

\begin{tabular}{l|c|c|c|c|c|c|c}
\hline $\begin{array}{l}\text { Correo electrónico } \\
\text { institucional }\end{array}$ & 3,98 & 1,05 & 821300 & $-3,837$ & 0,000 & Privada & 1905,82 \\
\hline Sitio web & 3,76 & 1,07 & 815804,5 & $-4,056$ & 0,000 & Pública & 1741,77 \\
\hline Facebook & 3,62 & 1,19 & & & & & \\
\hline Instagram & 3,03 & 1,36 & 723103 & $-8,068$ & 0,000 & Privada & 1914,69 \\
\hline YouTube & 2,94 & 1,37 & 888275,5 & $-0,749$ & 0,454 & & 1739,89 \\
\hline Twitter & 2,70 & 1,34 & 842119 & $-2,802$ & 0,005 & Privada & 2064,2 \\
\hline
\end{tabular}

Nivel de compromiso: nivel de acuerdo/desacuerdo con las siguientes afirmaciones Índice del nivel de compromiso universitario (INCU)

\begin{tabular}{l|l|l|l|l|l|l|l}
\hline $\begin{array}{l}\text { Recomendaría a otras } \\
\text { personas estudiar en esta } \\
\text { universidad }\end{array}$ & 4,19 & 1,01 & & & & & \\
\hline $\begin{array}{l}\text { Si pudiera volver a } \\
\text { elegir, elegiría la misma } \\
\text { universidad }\end{array}$ & 4,04 & 1,12 & 843453,5 & $-2,846$ & 0,004 & Privada & 1670,91 \\
\hline $\begin{array}{l}\text { La universidad me } \\
\text { envía información } \\
\text { oportunamente }\end{array}$ & 3,86 & 1,04 & 836623,5 & $-3,137$ & 0,002 & 1791,65 \\
\hline $\begin{array}{l}\text { Elegiría estudiar un } \\
\text { posgrado en la misma } \\
\text { universidad }\end{array}$ & 3,83 & 1,20 & 849755,5 & $-2,509$ & 0,012 & Privada & 1881,11 \\
\hline $\begin{array}{l}\text { La universidad se preocupa } \\
\text { por hacerme sentir } \\
\text { parte de la comunidad } \\
\text { universitaria }\end{array}$ & 3,72 & 1,13 & 840134,5 & $-2,941$ & 0,003 & Pública & 1747,01 \\
\hline $\begin{array}{l}\text { Me gusta compartir } \\
\text { información de la } \\
\text { universidad en mis redes } \\
\text { sociales }\end{array}$ & 3,45 & 1,15 & & & & & \\
\hline
\end{tabular}

Nota: los ítems de cada grupo de datos están ordenados de mayor a menor, en función de la media.

Tabla 4. Percepción como estudiante universitario

Fuente: Elaboración propia. 


\section{DISCUSIÓN Y CONCLUSIONES}

Las instituciones de educación superior en Ecuador, al conocer la percepción sobre la comunicación universitaria que tienen los estudiantes antes y después de ingresar a la universidad, podrán elaborar planificaciones de medios que sean más estratégicas, para fortalecer la relación con ellos.

Los resultados del estudio aplicado en este país demuestran que la dificultad de los estudiantes para elegir la universidad fue baja, especialmente para quienes decidieron estudiar en instituciones públicas. Durante el proceso de decisión, en conjunto influyeron en este orden, los factores institucionales, los de recomendación y los de marketing. De manera puntual, los tres primeros de los 20 factores que influyeron fueron los costos de estudios (factor institucional), la recomendación de padres/madres (factor de recomendación) y la localidad/ubicación de la universidad (factor institucional). Estos dos últimos coinciden con los resultados encontrados por Menon y sus colegas (2007) y por Simões y Soares (2010). Si bien los costos son dimensiones de carácter institucional y especialmente administrativo, la recomendación de padres/madres demuestra que las instituciones de educación superior deben valorarlos e incluirlos en sus planes de comunicación, como parte de sus audiencias prioritarias.

Los dos primeros factores de comunicaciones de marketing que más influyeron fueron las publicidades en redes sociales y las ferias de universidades. Durante el proceso de decisión, valoraron la información de los sitios web institucionales, como habían también mencionado Gibbs y Dean (2015) y en las visitas (Goodrich et al., 2018). Esto demuestra que para ayudar a tomar una correcta decisión, la comunicación de las universidades -más que exclusiva del entorno en línea u offline- debe ser integral, e importa contar con departamentos de comunicación propios y multidisciplinarios.

Para los estudiantes actuales es importante que sus universidades les mantengan al tanto sobre distintos temas, en el siguiente orden: de investigación, académicos, institucionales, de interés social y eventos. Sin embargo, a excepción de la investigación, el nivel de importancia que otorgan a cada tema difiere del nivel de satisfacción que sienten sobre cómo la institución maneja dichos temas. Esto podría indicar que la comunicación interna necesite mejorar.

Los estudiantes actuales valoran más los canales en línea. Consideran que los medios más convenientes para recibir información oportuna son las redes sociales y las plataformas académicas universitarias. Por otra parte, la frecuencia con que consultan seis canales en línea de sus universidades coincide con el nivel de satisfacción que sienten sobre cómo las IES los utilizan para dirigirse a ellos. En este caso, los que lideran la lista son el correo institucional y el sitio web. 
Considerando que las universidades ecuatorianas utilizan las redes sociales sobre todo para promocionar los programas de estudios a audiencias externas (SeguraMariño et al., 2020) los resultados sugieren que las IES deben incluir comunicación dirigida a sus estudiantes internos por este medio, e incluir temas de comunicación institucional en las plataformas académicas.

Como sugiere Helgesen (2008), la satisfacción influye en la lealtad de los estudiantes y puede evitar su deserción. En este estudio se analizó el compromiso que los alumnos sienten con sus universidades, mediante el índice del nivel de compromiso universitario (INCU) y cómo este compromiso podría llevar a la lealtad, pero para llegar a esa conclusión sería necesario realizar otro estudio. En este caso, se detectó que la satisfacción de temas y de canales institucionales están correlacionadas con el nivel de compromiso que los estudiantes sienten con sus universidades. Es decir, que cuanto más alta sea la satisfacción, más predisposición habrá para que, por ejemplo, recomienden estudiar en sus universidades, sientan que volverían a elegir la misma institución, piensen estudiar un posgrado en la misma institución, se sientan parte de la comunidad universitaria y compartan información en sus redes sociales. Cabe señalar que el nivel de compromiso no difiere según los tipos de universidades, pero sí el índice de satisfacción de temas y de canales institucionales, de modo que las privadas gestionan mejor la comunicación organizacional que las públicas.

No hubo mayores limitaciones para realizar esta investigación. En cuanto a la participación, hubo más disposición de la esperada para responder a la encuesta por parte del estudiantado, pero se desconoce si se logrará la misma respuesta en años posteriores con nuevas cohortes de estudiantes. Por ello, convendría llegar a acuerdos de colaboración entre instituciones de educación superior, los que también podrían propiciar una mayor participación de las autoridades universitarias en las investigaciones. En el caso de este estudio, ha resultado notablemente inferior a la esperada (se pretendía una participación de al menos 60\%). El ámbito de este estudio se ha limitado al análisis de las universidades ecuatorianas, si bien se sugiere realizar estudios similares en otros contextos geográficos, debido a las claras diferencias que existen entre países (Hemsley-Brown \& Oplatka, 2015).

Uno de los aspectos en los que difieren los sistemas de educación superior es en el tipo de financiamiento. Por eso, es recomendable simplificar en instituciones públicas y privadas para que se puedan comparar con resultados de otros estudios, en la medida de lo posible (Saraite-Sariene et al., 2018). Incluso existen diferencias en los detalles que caracterizan a dicha tipología: en España, Estados Unidos e Inglaterra las universidades públicas demandan un pago, a diferencia de países como Argentina, Finlandia, o Ecuador, entre otros, donde son gratuitas. 
Finalmente, se recomienda investigar sobre las necesidades de información y la percepción de padres/madres, así como evaluar qué tan informados están sobre la oferta de educación superior, ya que como se demostró en este estudio, ejercen una alta influencia en una de las decisiones más importantes de la vida de sus hijos.

\section{REFERENCIAS}

Aitken, N. (1982). College Student Performance, Satisfaction and Retention: Specification and Estimation of a Structural Model. Journal of Higher Education, 53(1), 32-50. https://doi.org/10.1080/00221546.1982.11780423

Alves, H. \& Raposo, M. (2010). The Influence of University Image on Student Behaviour. International Journal of Educational Management, 24(1), 73-85. https://doi.org/10.1108/09513541011013060

Berganza, R., Lavín, E., \& Piñeiro-Naval, V. (2017). La percepción de los periodistas españoles acerca de sus roles profesionales (Spanish journalists' perception about their professional roles). Comunicar, 51, 83-92. https://doi.org/10.3916/C51-2017-08

Bonilla, M. del Rocío, Perea, E., del Olmo, J. L., \& Corrons, A. (2020). Insights into user engagement on social media. Case study of a higher education institution. Journal of Marketing for Higher Education, 30(1), 145-160. https://doi.org/10.1080/08841241.2019.1693475

Carvalho, S. W. \& de Oliveira Mota, M. (2010). The Role of Trust in Creating Value and Student Loyalty in Relational Exchanges between Higher Education Institutions and Their Students. JournalofMarketingfor HigherEducation, 20(1),145-65. https://doi.org/10.1080/08841241003788201

Casidy, R. (2014). The role of perceived market orientation in the higher education sector. AustralasianMarketingJournal(AMJ), 22(2),155-163. https://doi.org/10.1016/j.ausmj.2014.02.001

Casidy, R. \& Wymer, W. (2018). A Taxonomy of Prestige-Seeking University Students: Strategic Insights for Higher Education. Journal of Strategic Marketing, 26(2), 140-55. https://doi.org/10.1080/0965254X.2016.1182573

Clark, M., Fine, M. B., \& Scheuer, C. L. (2017). Relationship Quality in Higher Education Marketing: The Role of Social Media Engagement. Journal of Marketing for Higher Education, 27(1), 40-58. https://doi.org/10.1080/08841241.2016.1269036

Constantinides, E. \& Zinck Stagno, M. C. (2011). Potential of the Social Media as Instruments of Higher Education Marketing: A Segmentation Study. Journal of Marketing for Higher Education, 21(1), 7-24. https://doi.org/10.1080/08841241.2011.573593

Deng, L. \& Chan, W. (2017). Testing the Difference Between Reliability Coefficients Alpha and Omega. Educational and Psychological Measurement, 77(2), 185-203. https://doi.org/10.1177/0013164416658325

Dunn, T. J., Baguley, T., \& Brunsden, V. (2014). From Alpha to Omega: A Practical Solution to the Pervasive Problem of Internal Consistency Estimation. British Journal of Psychology, 105(3), 399-412. https://doi.org/10.1111/bjop.12046

Elliott, K. M. \& Healy, M. A. (2001). Key Factors Influencing Student Satisfaction Related to Recruitment and Retention. Journal of Marketing for Higher Education, 10 (4), 1-11.

https://doi.org/10.1300/J050v10n04_01 
Erazo-Coronado, L., Llano-Aristizábal, S., Garcés-Prettel, M., \& Erazo-Coronado, A. M. (2020). Impacto de la comunicacion de la responsabilidad social universitaria en la seleccion de universidad y efecto mediador de la reputacion (Impact of university social responsibility communication on university selection and the mediating effect of reputation). El Profesional de la Información, 29(4). https://doi.org/10.3145/epi.2020.jul.38

Fayos, T., Gonzalez-Gallarza, M., Servera, D., \& Arteaga, F. (2011). Análisis y evaluación del servicio de formación universitaria: implicaciones para el marketing estratégico de las universidades (Analysis and assessment of the service of higher education: strategic marketing implications for universities). Revista de Investigación en Educación, 9(2), 133-52. Retrieved from http://reined.webs.uvigo.es/index.php/reined/article/view/119

Gallegos, J. A. \& Vasquez, A. (2019). Explaining university student loyalty: theory, method, and empirical research in Chile. Academia Revista Latinoamericana de Administración, 32(4), 525-540. https://doi.org/10.1108/ARLA-02-2019-0049

García-García, M. (2018). Universidad y medios sociales. Gestión de la comunicación en la universidad española (University and Social Media Management. Communication at the Spanish University). Prisma Social, (22), 20-36. Retrieved from https://revistaprismasocial.es/article/view/2535

Gibbs, P. \& Dean, A. (2015). Do Higher Education Institutes Communicate Trust Well? Journal of Marketing for Higher Education, 25(2), 155-70. https://doi.org/10.1080/08841241.2015.1059918

GlobalWebIndex. (2019). The Youth of the Nation: Global Trends Among Gen Z. Retrieved from https://assets.ctfassets.net/inb32lme5009/7wDIuSsLOnSxTUqPmRb081/ 603b8ffb77757549d39034884a23743c/The_Youth_of_the_Nations__Global_Trends_ Among_Gen_Z.pdf

Goodrich, K., Swani, K., \& Munch, J. (2018). How to connect with your best student prospects: saying the right things, to the right students, in the right media. Journal of Marketing Communications, 26(4), 434-453. https://doi.org/10.1080/13527266.2018.1514319

Hayes, T. J., Ruschman, D., \& Walker, M. M. (2009). Social Networking as an Admission Tool: A Case Study in Success. Journal of Marketing for Higher Education, 19(2), 109-124. https://doi.org/10.1080/08841240903423042

Helgesen, $\varnothing$. (2008). Marketing for Higher Education: A Relationship Marketing Approach.Journal of Marketing for Higher Education 18(1), 50-78. https://doi.org/10.1080/08841240802100188

Hemsley-Brown, J. \& Oplatka, I. (2015). University choice: what do we know, what don't we know and what do we still need to find out? International Journal of Educational Management, 29(3), 254-74. https://doi.org/10.1108/IJEM-10-2013-0150

Instituto de Estadística de la UNESCO. (2014). Campos de educación y capacitación 2013 de la CINE (ISCED-F 2013): Manual que acompaña la Clasificación Internacional Normalizada de la Educación 2011 (ISCED Fields of Education and Training 2013 (ISCED-F 2013): Manual accompanying the International Standard Classification of Education 2011). Montreal, Canada: Instituto de Estadística de la UNESCO. https://doi.org/10.15220/978-92-9189-157-3-sp

Khanna, M., Jacob, I., \& Yadav, N. (2014). Identifying and Analyzing Touchpoints for Building a Higher Education Brand. Journal of Marketing for Higher Education, 24(1), 122-43. https://doi.org/10.1080/08841241.2014.920460 
Kurniawan, Y., Setiawan, S., Bhutkar, G., \& Cabezas, D. (2020). Instagram Engagement for University. In 2020 International Conference on Information Management and Technology (ICIMTech) (pp. 887-892). IEEE. https://doi.org/10.1109/ICIMTech50083.2020.9211134

Maringe, F. (2006). University and course choice: Implications for positioning, recruitment and marketing. International Journal of Educational Management, 20(6), 466-79. https:// doi.org/10.1108/09513540610683711

Menon, M. E., Saiti, A., \& Socratous, M. (2007). Rationality, Information Search and Choice in Higher Education: Evidence from Greece. Higher Education, 54(5), 705-21. https://doi.org/10.1007/s10734-006-9019-3

Moogan, Y. J. (2011). Can a higher education institution's marketing strategy improve the student-institution match? International Journal of Educational Management, 25(6), 570-89. https://doi.org/10.1108/09513541111159068

Ortega, J. L. \& Aguillo, I. F. (2009). Mapping World-Class Universities on the Web. Information Processing \& Management, 45(2), 272-79. hthttps://doi.org/10.1016/j.ipm.2008.10.001

Özdemir, A., Tozlu, E., Şen, E., \& Ateşoğlu, H. (2016). Analyses of Word-of-Mouth Communication and Itseffect on Students' University Preferences. Procedia-Social and Behavioral Sciences, 235, 22-35. https://doi.org/10.1016/j.sbspro.2016.11.022

Peruta, A. \& Shields, A. B. (2016). Social Media in Higher Education: Understanding How Colleges and Universities Use Facebook. Journal of Marketing for Higher Education, 27(1), 131-43. https://doi.org/10.1080/08841241.2016.1212451

Rehman, M. A., Woyo, E., Akahome, J. E., \& Sohail, M. D. (2020). The influence of course experience, satisfaction, and loyalty on students' word-of-mouth and re-enrolment intentions. Journal of Marketing for Higher Education. https://doi.org/10.1080/08841241.2020.1852469

Rodríguez, A. \& Santamaría, P. (2012). Análisis del uso de las redes sociales en Internet: Facebook y Twitter en las Universidades españolas (Analysis of the use of social networks on the Internet: Facebook and Twitter in Spanish Universities). ICONO14, Revista científica de comunicación y tecnologías emergentes, 10(2), 228-246. https://doi.org/10.7195/ri14.v10i2.198

Rojas-Méndez, J. I., Vásquez-Párraga, A. Z., Kara, A., \& Cerda-Urrutia, A. (2009). Determinants of Student Loyalty in Higher Education: A Tested Relationship Approach in Latin America. Latin American Business Review, 10(1), 21-39. https://doi.org/10.1080/10978520903022089

Sampaio, C. H., Perin, M. G., Simões, C., \& Kleinowski, H. (2012). Students' Trust, Value and Loyalty: Evidence from Higher Education in Brazil. Journal of Marketing for Higher Education, 22(1), 83-100. https://doi.org/10.1080/08841241.2012.705796

Saraite-Sariene, L., Gálvez Rodríguez, M. D. M., \& Haro de Rosario, A. (2018). Exploring Determining Factors of Web Transparency in the World's Top Universities. Revista de Contabilidad, 21(1), 63-72. https://doi.org/10.1016/j.rcsar.2017.07.004

Segura-Mariño, A. G., Paniagua-Rojano, F. J., \& Fernández-Sande, M. (2020). Metodología para evaluar la comunicación universitaria en Facebook y Twitter (Methodology to evaluate university communication on Facebook and Twitter). Prisma Social, (28), 127-144. Retrieved from https://revistaprismasocial.es/article/view/3357 
Shields, A. B. \& Peruta, A. (2018). Social Media and the University Decision. Do Prospective Students Really Care? Journal of Marketing for Higher Education, 29(1), 67-83. https://doi.org/10.1080/08841241.2018.1557778

Simões, C. \& Soares, A. M. (2010). Applying to Higher Education: Information Sources and Choice Factors. Studies in Higher Education, 35(4). 371-389. https://doi.org/10.1080/03075070903096490

Slack, K., Mangan, J., Hughes, A., \& Davies, P. (2014). 'Hot', 'Cold’ and 'Warm' Information and Higher Education Decision-Making. British Journal of Sociology of Education, 35(2), 204-223. https://doi.org/10.1080/01425692.2012.741803

Sumartias, S. \& Nuraryo, I. (2017). Student satisfaction, University brand image and its impact on word of mouth communication. International Journal of Applied Business and Economic Research, 15(19), 475-483.

Sutherland, K., Davis, C., Terton, U., \& Visser, I. (2018). University Student Social Media Use and Its Influence on Offline Engagement in Higher Educational Communities. Student Success, 9(2), 13-24. https://doi.org/10.5204/ssj.v9i2.400

Vallaeys, F. (2008). Responsabilidad social universitaria: una nueva filosofía de gestión ética e inteligente para las universidades (University Social Responsibility: A New Philosophy of Ethical and Intelligent Management for Universities). Educación superior y sociedad, 13(2), 191-220. Retrieved from https://unesdoc.unesco.org/ark:/48223/pf0000182170

Veas, C. (2015). La Política y Gestión Comunicacional como aporte al desarrollo de la gestión institucional de las Universidades Chilenas (Communication Policy and Management as a contribution to Chilean universities institutional management). Revista Internacional de Relaciones Públicas, 5(9), 179-206. https://doi.org/10.5783/RIRP-9-2015-10-179-206

Vera Silva, C. \& Gouveia Rodrigues, R. (2012). Web Site Quality Evaluation in Higher Education Institutions. Procedia Technology, 5, 273-282. https://doi.org/10.1016/j.protcy.2012.09.030

Viladrich, C., Angulo-Brunet, A. \& Doval, D. (2017). Un viaje alrededor de alfa y omega para estimar la fiabilidad de consistencia interna (A journey around alpha and omega to estimate internal consistency reliability). Anales de Psicología, 33(3), 755-782.

https://doi.org/10.6018/analesps.33.3.268401 


\section{SOBRE LOS AUTORES}

ADRIANA GRACIELA SEGURA-MARIÑo, profesora de tiempo completo en la Escuela de Comunicación de la Pontificia Universidad Católica del Ecuador, Sede Santo Domingo. Sus principales líneas de investigación son: comunicación organizacional y comunicación online. Ha sido investigadora visitante en la Universidade da Beira Interior y en la Universidad Complutense de Madrid.

iD https://orcid.org/0000-0002-5071-1690

MANUEL FERNÁNDEZ-SANDE, profesor titular en el Departamento de Periodismo y Comunicación Global de la Universidad Complutense de Madrid. Sus principales líneas de investigación son: análisis del mercado radiofónico, podcasting, historia de la radio, dirección estratégica de empresas de comunicación y diversidad audiovisual. Ha sido investigador visitante en Glasgow Caledonian University, University of Cambridge y Universidade de São Paulo, entre otras. Actualmente dirige la revista Estudios sobre el Mensaje Periodístico y es coordinador adjunto del Doctorado en Periodismo de la Universidad Complutense.

FRANCISCO JAVIER PANIAGUA ROJANO, profesor titular en el Departamento de Periodismo de la Universidad de Málaga. Sus principales líneas de investigación son la comunicación estratégica, audiencias y profesión periodística y social media. Ha sido profesor visitante en la Universidad de Surrey. Actualmente es coordinador de la sección "Comunicación estratégica y organizacional" de la Asociación Española de Investigación de la Comunicación y vicedecano de Calidad e Innovación de la Facultad de Ciencias de la Comunicación de la Universidad de Málaga.

iD https://orcid.org/0000-0001-7376-4536 